\title{
Processing and Visualization of Diffusion MRI
}

\author{
James G. Malcolm ${ }^{1}$, Yogesh Rathi ${ }^{1}$, and Carl-Fredrik Westin ${ }^{2}$ \\ 1 Psychiatry Neuroimaging Laboratory, Harvard Medical School, Boston, MA \\ 2 Laboratory for Mathematics in Imaging, Harvard Medical School, Boston, MA
}

\begin{abstract}
This chapter provides a survey of techniques for processing and visualization of diffusion magnetic resonance imagery. We describe various approaches to modeling the local diffusion structure from scanner measurements. We then look at techniques to trace out neural pathways and infer global tissue structure. Last, we draw upon these as building blocks for the visualization and analysis of the neural architecture of individuals and groups.
\end{abstract}

\subsection{Introduction}

The advent of diffusion magnetic resonance imaging (dMRI) has provided the opportunity for non-invasive investigation of neural architecture. While structural MRI has long been used to image soft tissue and bone, dMRI provides additional insight into tissue microstructure by measuring its microscopic diffusion characteristics. To accomplish this, the magnetic field induces the movement of water while the presence of cell membranes, fibers, or other macromolecules hinder this movement. By varying the direction and strength of the magnetic fields, we essentially use the water molecules as a probe to get a sense of the local tissue structure.

At the lowest level this diffusion pattern provides several insights. For example, in fibrous tissue the dominant direction of allowed diffusion corresponds the underlying direction of fibers. In addition, quantifying the anisotropy of the diffusion pattern can also provide useful biomarkers. Several models have been proposed to interpret scanner measurements, ranging from geometric abstractions to those with biological motivation. In Sec. 18.2 we will introduce various models and methods for interpreting the diffusion measurements.

By connecting these local orientation models, tractography attempts to reconstruct the neural pathways. Tracing out these pathways, we begin to see how neurons originating from one region connect to other regions and how well-defined those connections may be. Not only can we examine properties 
of the local tissue but we begin to see the global functional architecture of the brain, but for such studies, the quality of the results relies heavily on the chosen fiber representation and the method of reconstructing pathways. In Sec. 18.3 we will describe several techniques for tracing out pathways.

At the highest level, neuroscientists can use the results of local modeling and tractography to examine individuals or groups of individuals. In Sec. 18.4 we will survey approaches to segment tissue with boundaries indistinguishable with structural MRI, apply network analysis to characterize the macroscopic neural architecture, reconstruct fiber bundles from individual fiber traces, and analyze groups of individuals.

\subsection{Modeling}

\subsubsection{Imaging the tissue}

The overall signal observed in an dMRI image voxel (millimetric) is the superposition of signals from many underlying molecules probing the tissue (micrometric). Thus, the image contrast is related to the strength of water diffusion. At each image voxel, diffusion is measured along a set of distinct gradients, $\mathbf{u}_{1}, \ldots, \mathbf{u}_{n} \in \mathbb{R}^{3}$, producing the corresponding signal, $\mathbf{s}=\left[s_{1}, \ldots, s_{n}\right]^{T} \in \mathbb{R}^{n}$. A general weighted formulation that relates the measured diffusion signal to the underlying fiber architecture may be written as,

$$
s_{i}=s_{0} \sum_{j} w_{j} e^{-b_{j} \mathbf{u}_{i}^{T} D_{j} \mathbf{u}_{i}},
$$

where $s_{0}$ is a baseline signal intensity, $b_{j}$ is the b-value, an acquisition-specific constant, $w_{j}$ are convex weights, and $D_{j}$ is a tensor describing a diffusion pattern. One of the first acquisition schemes developed, diffusion tensor imaging (DTI) utilizes these measurements to compute a Gaussian estimate of the diffusion orientation and strength at each voxel [13,22].

Going beyond this macroscopic description of diffusion, various higher resolution acquisition techniques have been developed to capture more information about the diffusion pattern. One of the first techniques, Diffusion Spectrum Imaging (DSI) measures the diffusion process at various scales by sampling densely throughout the voxel $[54,129]$. From this, the Fourier transform is used to convert the signal to a diffusion probability distribution. Due to the large number of samples acquired (usually more than 256 gradient directions), this scheme provides a much more accurate description of the diffusion process. However, on account of the large acquisition time (of the order of 1-2 hours per subject), this technique is not typically used in clinical scans and its use is restricted to few research applications.

Instead of spatially sampling the diffusion in a lattice throughout the voxel, a spherical shell sampling could be used [122]. Using this sampling technique, the authors in [123] demonstrated that the shape of the diffusion probability 
could be recovered from the images acquired on a single spherical shell. This significantly reduced the acquisition time, while providing most of the information about the underlying diffusion in the tissue. This naturally led to the application of techniques for estimating functions on a spherical domain. For example, Q-ball imaging (QBI) demonstrated a spherical version of the Fourier transform to reconstruct the probability diffusion as an isosurface [122].

To begin studying the microstructure of fibers with these imaging techniques, we need models to interpret these diffusion measurements. Such models fall broadly into two categories: parametric and nonparametric.

\subsubsection{Parametric models}

One of the simplest models of diffusion is a Gaussian distribution: an elliptic (anisotropic) shape indicating a strong diffusion direction while a more rounded surface (isotropic) indicating less certainty in any particular direction (see Fig. 1(c)). While robust, assuming this Gaussian model is inadequate in cases of mixed fiber presence or more complex orientations where the signal may indicate a non-Gaussian pattern $[6,46,125]$. To handle these complex patterns, higher resolution imaging and more flexible parametric models have been proposed including mixtures of tensors $[2,19,61,74,99,100,125]$ and directional functions $[68,88,107]$. While these typically require the number of components to be fixed or estimated separately, more continuous mixtures have also been proposed [65]. Further, biologically inspired models and tailored acquisition schemes have been proposed to estimate physical tissue microstructure $[9,10]$; see $[5]$ for more.

\subsubsection{Nonparametric models}

Nonparametric models can often provide more information about the diffusion pattern. Instead of modeling a discrete number of fibers as in parametric models, nonparametric techniques estimate a spherical orientation distribution function indicating potential fiber directions and the relative certainty thereof. For this estimation, an assortment of surface reconstruction methods have been introduced: Q-ball imaging to directly transform the signal into a probability surface [123], spherical harmonic representations $[7,37,46,58]$, higher-order tensors $[12,14,95]$, diffusion profile transforms [62,96], deconvolution with an assumed single-fiber signal response [64,120], and more. Fig. 1(e) shows a spherical harmonic reconstruction of the signal. Compare this to the original signal in Fig. 1(b).

It is important to keep in mind that there is a often distinction made between the reconstructed diffusion orientation distribution function and the putative fiber orientation distribution function; while most techniques estimate the diffusion function, its relation to the underlying fiber function is still an open problem. Spherical convolution is designed to directly transform the 


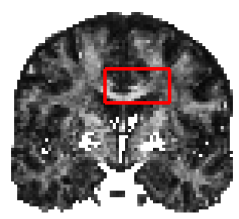

(a) Slice indicating region of interest

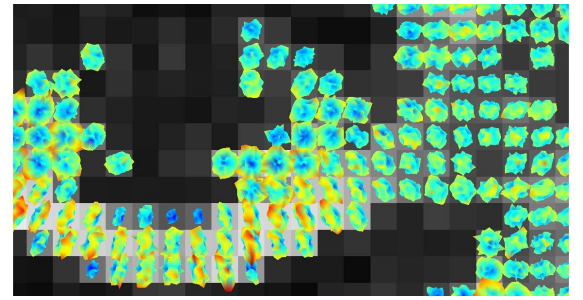

(b) Original signal

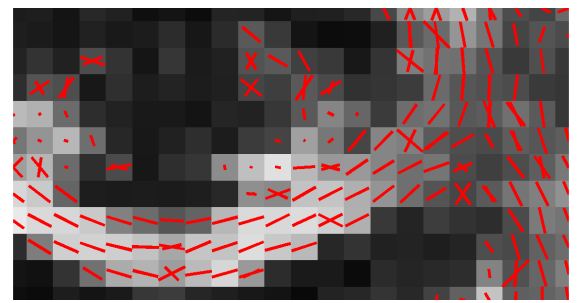

(d) Two-tensor axes

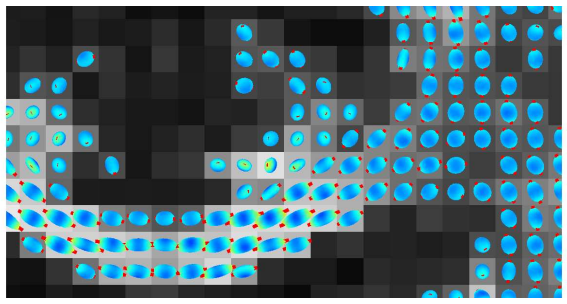

(c) Single-tensor with axis

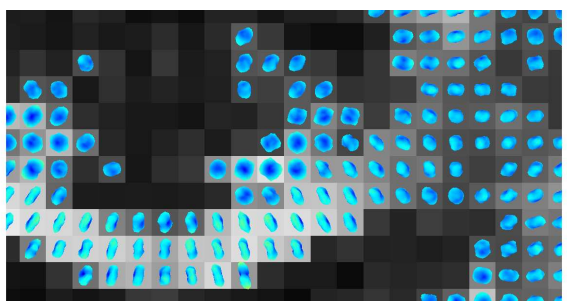

(e) Spherical harmonic signal

Fig. 18.1. Comparison of various models within a coronal slice (a) passing through the corpus callosum. In (b) the original signal appears noisy. In (c) a single tensor fit provides a robust estimate of the principal diffusion direction. In (d) a two-tensor model is fit to planar voxels and the two axes are reported [100]. In (e) spherical harmonics provide a smoothed non-parametric estimate of the signal surface eliminating much of the noise seen in (b) [37].

signal into a fiber distribution $[4,7,62,120]$, yet diffusion sharpening strategies have been developed to deal with Q-ball and diffusion functions [38].

While parametric methods directly describe the principal diffusion directions, interpreting the diffusion pattern from model independent representations typically involves determining the number and orientation of principal diffusion directions present. A common technique is to find them as surface maxima of the diffusion function $[24,38,58,120]$, while another approach is to decompose a high-order tensor representation of the diffusion function into a mixture of rank-1 tensors [115]. 


\subsubsection{Regularization}

As in all physical systems, the measurement noise plays a nontrivial role, and so several techniques have been proposed to regularize the estimation. One could start by directly regularizing the MRI signal by designing filters based on the various signal noise models $[1,11,71]$. Alternatively, one could estimate the diffusion tensor field and then correct these estimated quantities [121]. For spherical harmonic modeling, a regularization term can be been directly included in the least squares formulation [37,58]. Attempts such as these to manipulate diffusion weighted images or tensor fields have received considerable attention regarding appropriate algebraic and numeric treatments [17, 44, 70,121].

Instead of regularizing signal or model parameters directly, an alternative approach is to infer the underlying geometry of the vector field [112]. Another interesting approach treats each newly acquired diffusion image as a new system measurement. Since diffusion tensors and spherical harmonics can be estimated within a least-squares framework, one can use a Kalman filter to update the estimate and optionally stop the scan when the model parameters converge [105]. Further, this online technique can be used to alter the gradient set so that, were the scan to be stopped early, the gradients up to that point are optimally spread (active imaging) [35].

\subsubsection{Characterizing tissue}

The goal of diffusion imaging is to draw inferences from the diffusion measurements. As a starting point, one often converts the diffusion weighted image volumes to a scalar volume much like structural MRI or CT images. Starting with the standard Gaussian diffusion tensor model, an assortment of scalar measures have been proposed to quantify the size, orientation, and shape of the diffusion pattern $[15,130]$. For example, fractional anisotropy quantifies the deviation from an isotropic tensor, an appealing quantity because it corresponds to the strength of diffusion while remaining invariant to orientation. Derivatives of these scalar measures have also been proposed to capture more information about the local neighborhood $[69,114]$, and these measures have been extended to high-order tensors [97]. Further, a definition of generalized anisotropy has been proposed to directly characterize anisotropy in terms of variance in the signal, hence avoiding an assumed model [125]. While geometric in nature, studies have shown these to be reasonable proxy measures for neural myelination $[18,66,91]$. Some studies have also examined the sensitivity of such measures against image acquisition schemes [29,131].

Meaningful visualization of diffusion images is difficult because of their multivariate nature, and much is lost when reducing the spectral signal down to scalar intensity volumes. Several geometric abstractions have been proposed to convey more information. Since the most common voxel model is 


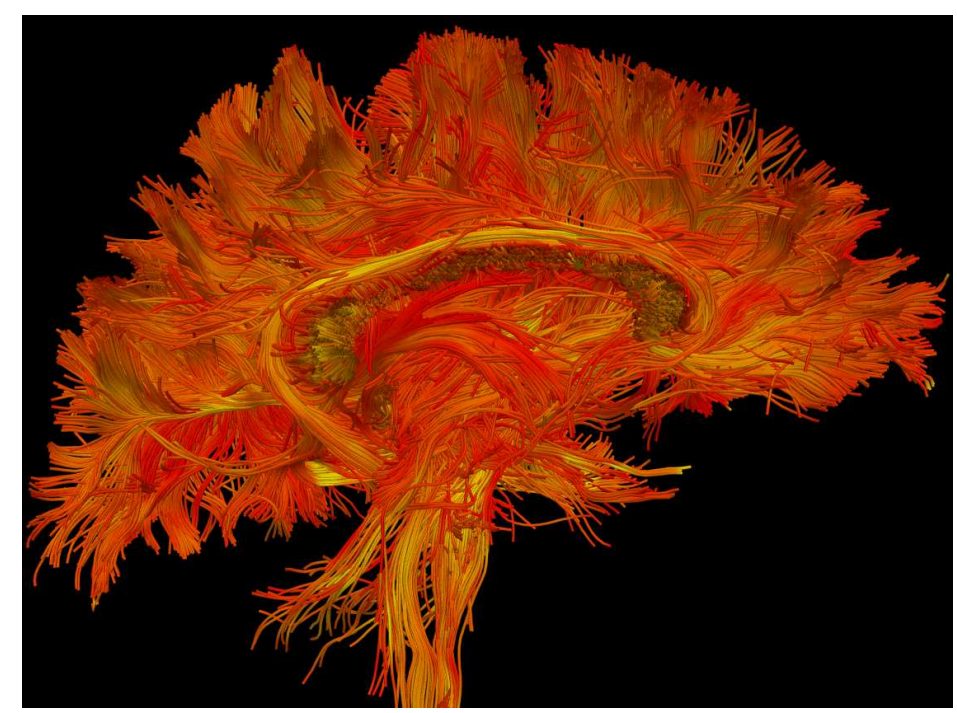

Fig. 18.2. Cutaway showing tractography throughout the left hemisphere colored by FA to indicate diffusion strength [87]. From this view, the fornix and cingulum bundle are visible near the center.

still the Gaussian diffusion tensor, most of the effort has focused on visualizing this basic element. The most common glyph is an ellipsoid simultaneously representing the size, shape, and orientation; however, since tensors have six free parameters, more elaborate representations have been proposed to visualize these additional dimensions using color, shading, or subtle variations in shape $[42,70,126,130]$. Apart from tensors, visualization strategies for other models have received comparatively little attention, the typical approach being to simply to visualize the diffusion isosurface at each voxel.

A vast literature exists on methods of acquisition, modeling, reconstruction, and visualization of diffusion images. For a comprehensive view, we suggest $[3,36,54,89,122,130]$.

\subsection{Tractography}

To compliment the wide assortment of techniques for signal modeling and reconstruction, there is an equally wide range of techniques to infer neural pathways. At the local level, one may categorize them either as tracing individual connections between regions or as diffusing out to estimate the probability of connection between regions. In addition, more global approaches have been developed to consider, not just the local orientations, but the suitability of entire paths when inferring connections. 

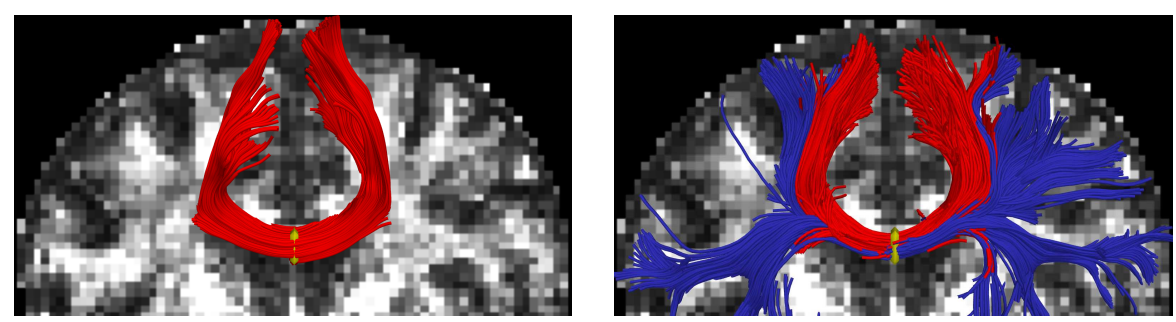

Fig. 18.3. Tractography from the center of the corpus callosum (seed region in yellow). The single-tensor model (left) captures only the corona radiata and misses the lateral pathways known to exist. The two-tensor method [87] (right) reveals many of these missing pathways (highlighted in blue).

\subsubsection{Deterministic tractography}

Deterministic tractography involves directly following the diffusion pathways. Typically, one places several starting points (seeds) in one region of interest and iteratively traces from one voxel to the next, essentially path integration in a vector field. One terminates these fiber bundles when the local diffusion appears week or upon reaching a target region. Fig. 18.2 offers a glimpse from inside the brain using this basic approach. Often additional regions are used as masks to post-process results, e.g. pathways from region A but not touching region $\mathrm{B}$.

In the single tensor model, standard streamline tractography follows the principal diffusion direction of the tensor [90], while multi-fiber models often include techniques for determining the number of fibers present or when pathways branch $[53,57,74]$. Since individual voxel measurements may be unreliable, several techniques have been developed for regularization. For example, using the estimate from the previous position $[78,134]$ as well as filtering formulations for path regularization [52] and model-based estimation [87]. The choice of model and optimization mechanism can drastically effect the final tracts. To illustrate, Fig. 18.3 shows tractography from the center of the corpus callosum using a single-tensor model and a two-tensor model using the filtered technique from [87].

\subsubsection{Probabilistic tractography}

While discrete paths intuitively represent the putative fiber pathways of interest, they tend to ignore the inherent uncertainty in estimating the principle diffusion directions in each voxel. Instead of tracing discrete paths to connect voxels, one may instead query the probability of voxel-to-voxel connections given the diffusion probability distributions reconstructed in each voxel.

Several approaches have been developed based on sampling. For example, one might run streamline tensor tractography treating each as a Monte Carlo sample; the more particles that take a particular path, the more likely 
that particular fiber pathway $[20,23,72,98]$. Another approach would be to consider more of the continuous diffusion field from Q-ball or other reconstructions $[16,48,99,101,124,133]$. By making high curvature paths unlikely, path regularization can be naturally enforced within the probabilistic framework. Another approach is to propagate an arrival-time isosurface from the seed region out through the diffusion field, the front evolution force being a function of the local diffusivity $[16,28,119]$.

Using the full diffusion reconstruction to guide particle diffusion has the advantage of naturally handling uncertainty in diffusion measurements, but for that same reason it tends toward diffuse tractography and false-positive connections. One option is to constrain diffusivity by fitting a model, thereby ensuring definite diffusion directions yet still taking into account some uncertainty $[20,48,99]$. A direct extension is to introduce a model selection mechanism to allow for additional components where appropriate [19,47]. However, one could stay with the nonparametric representations and instead sharpen the diffusion profile to draw out the underlying fiber orientations $[38,118]$.

\subsubsection{Global tractography}

Despite advances in voxel modeling, discerning the underlying fiber configuration has proven difficult. For example, looking at a single voxel, the symmetry inherent in the diffusion measurements makes it difficult to tell if the observed pattern represents a fiber curving through the voxel or instead represents a fanning pattern. Reliable and accurate fiber resolution requires more information than that of a single voxel. For example, instead of estimating the fiber orientation, one could instead infer the geometry of the entire neighborhood [113].

Going a step further, one could say that reliable and accurate connectivity resolution requires even more information, beyond simply a voxel neighborhood. In some respects, probabilistic tractography can be seen to take into account more global information. By spawning thousands of particles, each attempting to form an individual connection, probabilistic techniques are able to explore more possibilities before picking those that are likely [98]. However, if these particles still only look at the local signal as they propagate from one voxel to the next, then they remain susceptible to local regions of uncertainty. Even those with resampling schemes are susceptible since the final result is still a product of the method used in local tracing $[23,133]$.

A natural step to address such problems is to introduce global connectivity information into local optimization procedures of techniques mentioned above. The work of [63] does this by extending the local Bayesian formulation in [19] with an additional prior that draws upon global connectivity information in regions of uncertainty. Similarly, one could use an energetic formulation still with data likelihood and prior terms, but additionally introduce terms governing the number of components present [43]. 
Another approach is to treat the entire path as the parameter to be optimized and use global optimization schemes. For example, one could model pathways as piecewise linear with a data likelihood term based on signal fit and a prior on spatial coherence of those linear components [73,109]. One advantage of this path-based approach is that it somewhat obviates the need for a multi-fiber voxel model; however, such a flexible global model dramatically increases the computational burden.

An alternative formulation is to find geodesic paths through the volume. Again using some form of data likelihood term, such methods then employ techniques for front propagation to find globally optimal paths of connection $[45,80,92,102,106]$.

Tractography is often used in group studies which typically require a common atlas for inter-subject comparison. Beginning with the end in mind, one could determine a reference bundle as a template and use this to drive tractography. This naturally ensures both the general geometric form of the solution and a direct correspondence between subjects $[41,51]$. Alternatively, the tract seeding and other algorithm parameters could be optimized until the tracts (data driven) approach the reference (data prior) [30]. Since this requires prespecifying such a reference bundle, information that may be unavailable or difficult to obtain, one could even incorporate the formulation of the reference bundle into the optimization procedure itself [31].

\subsubsection{Validation}

In attempting to reconstruct neural pathways virtually, it is important to keep in mind the inherent uncertainty in such reconstructions. The resolution of dMRI scanners is at the level of $3-10 \mathrm{~mm}^{3}$ while physical fiber axons are often an order of magnitude smaller in diameter-a relationship which leaves much room for error. Some noise or a complex fiber configuration could simply look like a diffuse signal and cause probabilistic tractography to stop in its tracks, while a few inaccurate voxel estimations could easily send the deterministic tracing off course to produce a false-positive connection. Even global methods could produce a tract that fits the signal quite well but incidentally jumps over an actual boundary in one or two voxels it thinks are noise. Consequently, a common question is: Are these pathways really present?

With this in mind, an active area of study is validating such results. Since physical dissection often requires weeks of tedious effort, many techniques have been used for validating these virtual dissections. A common starting point is to employ synthetic and physical phantoms with known parameters when evaluating new methods [104]. When possible, imaging before and after injecting radio-opaque dyes directly into the tissue can provide some of the best evidence for comparison [33,82]. Another powerful approach is to apply bootstrap sampling or other non-parametric statistical tests to judge the sensitivity and reproducibility of resulting tractography against algorithm parameters, image acquisition, and even signal noise $[29,30,49,67,77,131]$. 


\subsection{Applications}

Having outlined various models and methods of reconstructing pathways, we now briefly cover several methods of further analysis.

\subsubsection{Volume segmentation}

Medical image segmentation has a long history, much of it focused on scalar intensity-based segmentation of anatomy. For neural segmentation, structural MRI easily reveals the boundaries between gray-matter and white-matter, and anatomic priors have helped further segment some internal structures [103]; however, the boundaries between many structures in the brain are remain invisible with structural MRI alone. The introduction of dMRI has provided new discriminating evidence in such cases where tissue may appear homogeneous on structural MRI or CT but contain distinct fiber populations.

To begin, most work has focused segmentation of the estimated tensor fields. Using suitable metrics to compare tensors, these techniques often borrow directly from active contour or graph cut segmentation with the approach of separating distributions. For example, one could define a Gaussian distribution of tensors to approximate a structure of interest [34,111]. For tissues with more heterogeneous fiber populations, e.g. the corpus callosum as it bends, such global parametric representations are unsuitable. For this, nonparametric approaches are more appropriate at capturing the variation throughout such structures $[84,108]$. Another approach to capture such variation is to limit the parametric distributions to local regions of support, essentially robust edge detection [76].

In Fig. 18.4 we see a graph cut segmentation of the corpus callosum [84]. The color-coded fractional anisotropy image is shown for visualization while segmentation was performed on the underlying tensor data. If statistics are computing ignoring the tensor manifold (Euclidean assumption), the final segmentation fails Fig. 4(b). If statistics are computing via a Riemannian mapping that respects this structure, the final segmentation is accurate Fig. 4(c). This highlights the need for appropriate algebraic treatment of tensors and other non-Euclidean models.

An altogether different approach to segmenting a structure is to divide it up according to where portions connect elsewhere. For example, the thalamus contains several nuclei indistinguishable in standard MR or even with contrast. After tracing connections from the thalamus to the cortex, one study demonstrated that grouping these connections revealed the underlying nuclei [21].

\subsubsection{Fiber clustering}

The raw output of full-brain tractography can produce hundreds of thousands of such tracings, an overwhelming amount of information. One approach to 


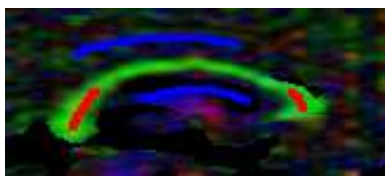

(a) Initial seed regions

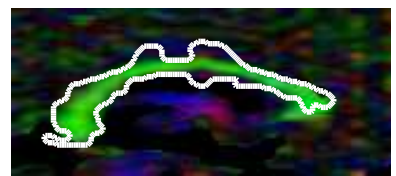

(b) Euclidean mapping

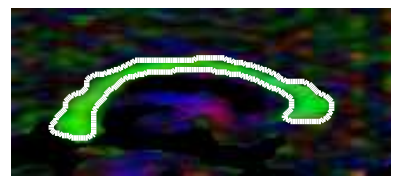

(c) Riemannian mapping

Fig. 18.4. Segmenting the corpus callosum using the graph cut technique from [84] (side view). The Euclidean mapping (b) does not take into account the structure of the underlying tensor manifold. The Riemannian mapping (c) takes this structure into account when computing statistics and so produces a correct segmentation.

understanding and visualizing such results is to group individual tracings into fiber bundles. Such techniques are typically based around two important design choices: the method of comparing fibers, and the method of clustering those fibers.

In comparing two fibers, one often starts by defining a distance measure, these typically being based on some point-to-point correspondence between the fibers $[32,39,93]$. With this correspondence in hand, one of the most common distances is then to take the mean closest point distance between the two fibers (Hausdorff distance). An alternative is to transform each fiber to a new vector space with a natural norm, e.g. a fiber of any length can be encoded with only the mean and covariance of points along its path and then use the $\mathrm{L}_{2}$ distance [25]. An altogether different approach is to consider the spatial overlap between fibers $[127,128]$. Since full-brain tractography often contains many small broken fragments as it tries to trace out bundles, such fragments are often separated from their actual cluster. Measures of spatial overlap may be more robust in such cases. In each of these methods, fibers were only considered as sequences of points, i.e. connections and orientations were ignored. Recent work demonstrates that incorporating such considerations provides robust descriptors of fiber bundles [40].

Based on these distances, several methods have been developed to cluster the fibers. Spectral methods typically begin with the construction of a Gram matrix encoding the pairwise affinity between any two fibers. After which normalized cuts can be applied to partition the Gram matrix and hence the fibers [25]. Affinity propagation has recently been demonstrated as an efficient and robust alternative which automatically determines the number of clusters to support a specified cluster size preference [79,86]. In Fig. 18.5 shows how clustering can automatically reveal known structures and provide a more coherent view of the brain. In addition, clustering can be used to judge outliers. For example, Fig. 18.6 reveals several streamlines that appear to have gone off track relative to the cluster centers.

Another clustering approach is to use the inner product space itself. For example, one can efficiently group directly on the induced manifold by iteratively joining fibers most similar until the desired clustering emerges [128]. 

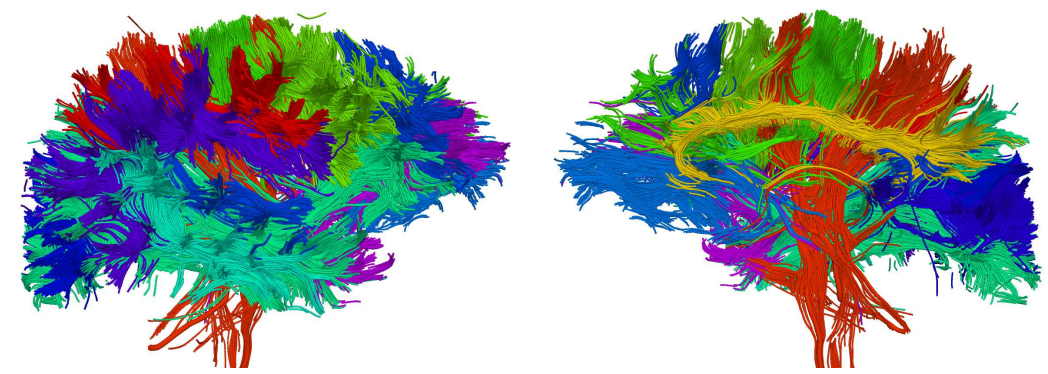

Fig. 18.5. Full-brain streamline tractography clustered using affinity propagation [86]. Viewed from the outside (left) and inside cutting away the left hemisphere (right). Among the visible structures, we see the cingulum bundle (yellow), internal capsule (red), and arcuate (purple).

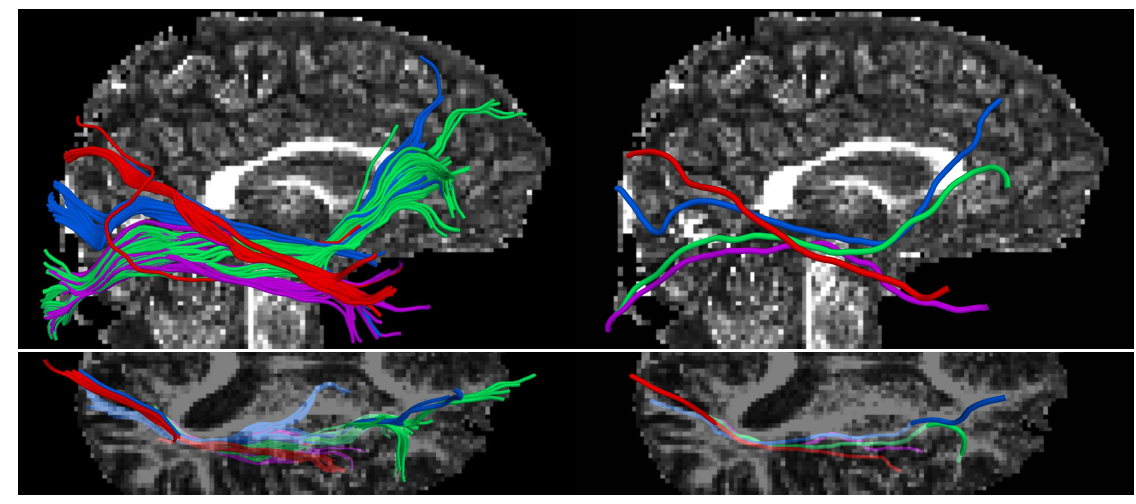

Fig. 18.6. Fronto-occipital fibers from the right hemisphere using streamline tractography and clustered (left) [86]. Viewing the cluster centers (right) we see several fibers (red) that appear to have wandered off the pathway.

To avoid construction of the large Gram matrix, variants of expectation maximization have been demonstrated to iteratively cluster fibers, an approach that naturally lends itself to incorporating anatomic priors [83, 93, 127]. Alternatively, one can begin with the end in mind by registering a reference fiber bundle template to patients thus obviating any need for later spatial normalization or correspondence [31].

\subsubsection{Connectivity}

While tissue segmentation can provide global cues of neural organization, it tells little of the contribution of individual elements. Similarly, while clustered tracings are easily visualized, deciphering the flood of information from fullbrain tractography demands more comprehensive quantitative analysis. For this, much has been borrowed from network analysis to characterize the neural 


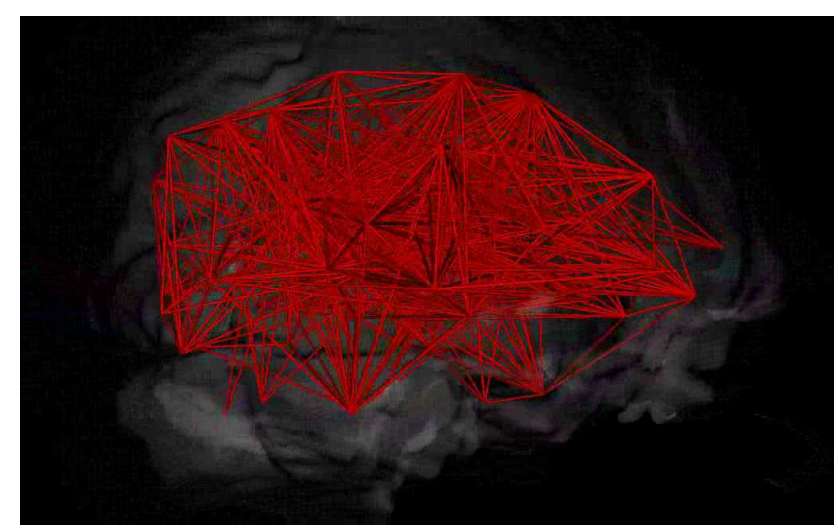

Fig. 18.7. The brain viewed as a network of weighted connections. Each edge represents a possible connection and is weighted by the strength of that path. Many techniques from network analysis are applied to reveal hubs and subnetworks within this macroscopic view.

topology. To start, instead of segmenting fibers into bundles, one can begin by classifying voxels into hubs or subregions into subnetworks [50, $55,56,117]$.

Dividing the brain up into major functional hubs, one can then view it as a graphical network as in Fig. 18.7. Each of these edges is then often weighted as a function of connection strength [56], but may also incorporate functional correlation to give further evidence of connectivity.

One of the first results of such analysis was the discovery of dense hubs linked by short pathways, a characteristic observed in many complex physical systems (small-world phenomena). Another interesting finding came from combining anatomic connections from dMRI with neuronal activity provided by fMRI [59]. They found that areas which are functionally connected are often not structurally connected, hence tractography alone does not provide the entire picture.

For a recent review of this emerging field of structural and functional network analysis, we recommend [26].

\subsubsection{Tissue analysis}

In forming population studies, there are several approaches for framing the analysis among patients. For example, voxel-based studies examine tissue characteristics in regions of interest [8]. Discriminant analysis has been applied to determine such regions [27]. Alternatively, one could also perform regression on the full image volume taking into account not only variation in diffusion but also in the full anatomy [110]. In contrast, tract-based studies incorporate the results of tractography to use fiber pathways as the frame of reference $[39,116]$, and several studies have demonstrated the importance of taking into account local fluctuations in estimated diffusion $[32,51,83,94,132]$. 


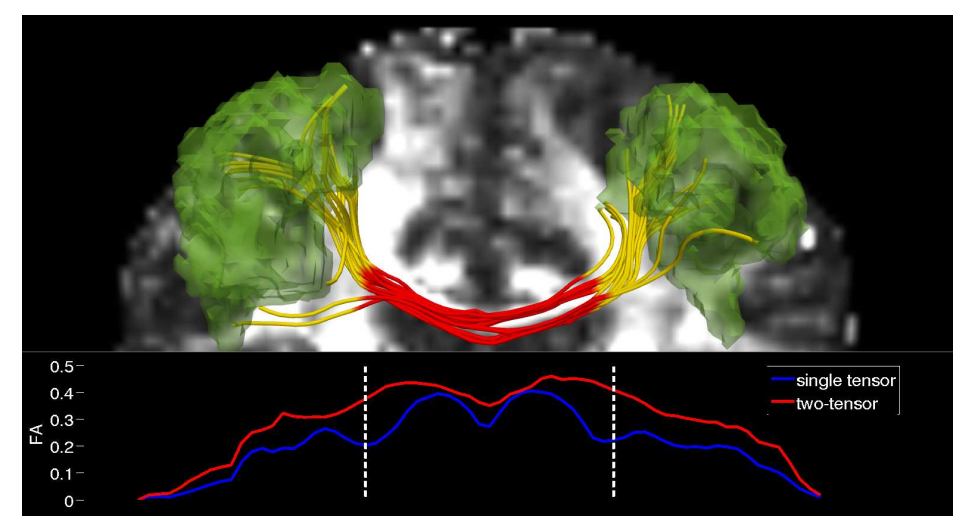

Fig. 18.8. Plotting FA as a function of arc-length to examine local fluctuations. Fibers are selected that connect the left and right seed regions (green). Notice how the FA from single-tensor (blue) is lower in regions of crossing compared to twotensor FA (red).

A common approach in many of these studies is to focus on characterizing individual pathways or bundles. To illustrate this analysis, Fig. 18.8 shows fibers connecting a small region in each hemisphere. We then average FA plotted along the bundle as a function of arc-length. Further, we plot the FA from both single- and two-tensor models to show how different models often produce very different tissue properties [85].

Several reviews exist documenting the application and findings of using various methods $[60,75,81]$.

\subsection{Summary}

Diffusion MRI has provided an unprecedented view of neural architecture. With each year, we develop better image acquisition schemes, more appropriate diffusion models, more accurate pathway reconstruction, and more sensitive analysis.

In this survey, we began with an overview of the various imaging techniques and diffusion models. While many acquisition sequences have become widely distributed for high angular resolution imaging, work continues in developing sequences and models capable of accurate resolution of biological properties such as axon diameter and degree of myelination $[5,10]$. We then reviewed various parametric models starting with the diffusion tensor on up to various mixture models as well as high-order tensors. Work continues to develop more accurate and reliable model estimation by incorporating information from neighboring voxels [87,113]. Further, scalar measures derived from these models similarly benefit from incorporating neighborhood information [114]. 
Next we outlined various methods of tractography to infer connectivity. Broadly, these techniques took either a deterministic or probabilistic approach. We also documented the recent trend toward global approaches, those that combine local voxel-to-voxel tracing with a sense of the full path $[43,109]$. Even with such considerations, tractography is has proven quite sensitive to image acquisition and initial conditions, so much work has gone into validation. Common techniques are the use of physical phantoms [104] or statistical tests like bootstrap analysis $[30,67,77]$.

Finally, we briefly introduced several machine learning approaches to make sense of the information found in diffusion imagery. Starting with segmentation, several techniques for scalar intensity segmentation have been extended

to dMRI. With the advent of full-brain tractography providing hundreds of thousands of fiber paths, the need to cluster connections into bundles has become increasingly important. The application of network analysis to connectivity appears to be an emerging area of research, especially in combination with alternate imaging modalities [26]. Finally, we noted several approaches to the analysis of neural tissue itself in regions of interest or along pathways.

\section{References}

1. S. Aja-Fernandez, M. Niethammer, M. Kubicki, M. E. Shenton, and C.-F. Westin. Restoration of DWI data using a Rician LMMSE estimator. Trans. on Med. Imag., 27:1389-1403, 2008.

2. A. Alexander, K. Hasan, J. Tsuruda, and D. Parker. Analysis of partial volume effects in diffusion-tensor MRI. Magnetic Resonance in Medicine, 45:770-780, 2001.

3. D. C. Alexander. An introduction to computational diffusion MRI: the diffusion tensor and beyond. In J. Weichert and H. Hagen, editors, Visualization and Image Processing of Tensor Fields, chapter 5. Springer, 2005.

4. D. C. Alexander. Maximum entropy spherical deconvolution for diffusion MRI. In Information Processing in Medical Imaging (IPMI), pages 76-87, 2005.

5. D. C. Alexander. Modelling, fitting and sampling in diffusion MRI. In Visualization and Processing of Tensor Fields, chapter 1, pages 3-20. Springer, 2009.

6. D. C. Alexander, G. Barker, and S. Arridge. Detection and modeling of nonGaussian apparent diffusion coefficient profiles in human brain data. Magnetic Resonance in Medicine, 48:331-340, 2002.

7. A. Anderson. Measurement of fiber orientation distributions using high angular resolution diffusion imaging. Magnetic Resonance in Medicine, 54(5):11941206, 2005.

8. J. Ashburner and K. Friston. Voxel-based morphometry-The methods. NeuroImage, 11(6):805-821, 2000.

9. Y. Assaf and P. Basser. Composite hindered and restricted model of diffusion (CHARMED) MR imaging of the human brain. NeuroImage, 27:48-58, 2005.

10. Y. Assaf, T. Blumenfeld-Katzir, Y. Yovel, and P. J. Basser. AxCaliber: A method for measuring axon diameter distribution from diffusion MRI. Magnetic Resonance in Medicine, 59:1347-1354, 2008. 
11. H.-E. Assemlal, David Tschumperlé, and L. Brun. Robust variational estimation of PDF functions from diffusion MR signal. In Workshop on Computational Diffusion MRI, 2008.

12. A Barmpoutis, M S Hwang, D Howland, J Forder, and B Vemuri. Regularized positive-definite fourth order tensor field estimation from DW-MRI. Neuroimage, 2009 .

13. P. Basser, J. Mattiello, and D. LeBihan. Estimation of the effective selfdiffusion tensor from the NMR spin echo. J. of Magnetic Resonance, 103:247254, 1994

14. P. J. Basser and S. Pajevic. Spectral decomposition of a $4^{\text {th }}$-order covariance tensor: Applications to diffusion tensor MRI. Signal Processing, 87:220-236, 2007.

15. P. J. Basser and C. Pierpaoli. Microstructural features measured using diffusion tensor imaging. J. of Magnetic Resonance, 111:209-219, 1996.

16. P. G. Batchelor, D. L. Hill, F. Calamante, and D. Atkinson. Study of connectivity in the brain using the full diffusion tensor from MRI. In Information Processing in Medical Imaging (IPMI), pages 121-133, 2001.

17. P. G. Batchelor, M. Moakher, D. Atkinson, F. Calamante, and A. Connelly. A rigorous framework for diffusion tensor calculus. Magnetic Resonance in Medicine, 53(1):221-225, 2005.

18. C. Beaulieu. The basis of anisotropic water diffusion in the nervous system-A technical review. NMR in Biomedicine, 15:438-455, 2002.

19. T. Behrens, H. Johansen-Berg, S. Jbabdi, M. Rushworth, and M. Woolrich. Probabilistic diffusion tractography with multiple fibre orientations: What can we gain? NeuroImage, 34:144-155, 2007.

20. T. Behrens, M. Woolrich, M. Jenkinson, H. Johansen-Berg, R. Nunes, S. Clare, P. Matthews, J. Brady, and S. Smith. Characterization and propagation of uncertainty in diffusion-weighted MR imaging. Magnetic Resonance in Medicine, 50:1077-1088, 2003.

21. T. E. J. Behrens, H. Johansen-Berg, M. W. Woolrich, S. M. Smith, C. A. M. Wheeler-Kingshott, P. A. Boulby, G. J. Barker, E. L. Sillery, K. Sheehan, O. Ciccrelli, A. J. Thompson, J. M. Brady, and P. M. Mathews. Non-invasive mapping of connections between human thalamus and cortex using diffusion imaging. Nature Neuroscience, 6(7):750-757, 2003.

22. D. Le Bihan. Looking into the functional architecture of the brain with diffusion MRI. Nature Reviews Neuroscience, 4:469-480, 2003.

23. M. Björnemo, A. Brun, R. Kikinis, and C.-F. Westin. Regularized stochastic white matter tractography using diffusion tensor MRI. In Medical Image Computing and Computer Assisted Intervention (MICCAI), pages 435-442, 2002.

24. L. Bloy and R. Verma. On computing the underlying fiber directions from the diffusion orientation distribution function. In Medical Image Computing and Computer Assisted Intervention (MICCAI), pages 1-8, 2008.

25. A. Brun, H. Knutsson, H. J. Park, M. E. Shenton, and C.-F. Westin. Clustering fiber traces using normalized cuts. In Medical Image Computing and Computer Assisted Intervention (MICCAI), pages 368-375, 2004.

26. E. Bullmore and O. Sporns. Complex brain networks: graph theoretical analysis of structural and functional systems. Nature Reviews Neuroscience, 10:186-198, 2009 . 
27. M. Caan, K. Vermeer, L. van Vliet, C. Majoie, B. Peters, G. den Heeten, and F. Vos. Shaving diffusion tensor images in discriminant analysis: A study into Schizophrenia. Medical Image Analysis, 10(6):841-849, 2006.

28. J. S. W. Campbell, K. Siddiqi, V. V. Rymar, A. F. Sadikot, and G. B. Pike. Flow-based fiber tracking with diffusion tensor and Q-ball data: Validation and comparison to principal diffusion direction techniques. NeuroImage, 27(4):725736, 2005.

29. S. Chung, Y. Lu, and R. Henry. Comparison of bootstrap approaches for estimation of uncertainties of DTI parameters. NeuroImage, 33(2):531-541, 2006.

30. J. Clayden, A. Storkey, and M. Bastin. A probabilistic model-based approach to consistent white matter tract segmentation. Trans. on Med. Imag., 26(11):1555-1561, 2007.

31. J. D. Clayden, A. J. Storkey, S. M. Maniega, and M. E. Bastin. Reproducibility of tract segmentation between sessions using an unsupervised modelling-based approach. NeuroImage, 45:377-385, 2009.

32. I. Corouge, P. T. Fletcher, S. Joshi, S. Gouttard, and G. Gerig. Fiber tractoriented statistics for quantitative diffusion tensor MRI analysis. Medical Image Analysis, 10(5):786-798, 2006.

33. J. Dauguet, S. Peled, V. Berezovskii, T. Delzescaux, S. K. Warfield, R. Born, and C.-F. Westin. Comparison of fiber tracts derived from in-vivo DTI tractography with $3 \mathrm{D}$ histological neural tract tracer reconstruction on a macaque brain. NeuroImage, 37:530-538, 2007.

34. R. de Luis-Garcia and C. Alberola-Lopez. Mixtures of Gaussians on tensor fields for the segmentation of DT-MRI. In Medical Image Computing and Computer Assisted Intervention (MICCAI), pages 319-326, 2007.

35. R. Deriche, J. Calder, and M. Descoteaux. Optimal real-time Q-Ball imaging using regularized Kalman filtering with incremental orientation sets. Medical Image Analysis, 13(4):564-579, 2009.

36. M. Descoteaux. High Angular Resolution Diffusion MRI: from Local Estimation to Segmentation and Tractography. PhD thesis, University of Nice-Sophia Antipolis, 2008.

37. M. Descoteaux, E. Angelino, S. Fitzgibbons, and R. Deriche. Regularized, fast, and robust analytical Q-ball imaging. Magnetic Resonance in Medicine, 58:497-510, 2007.

38. M. Descoteaux, R. Deriche, T. Knoesche, and A. Anwander. Deterministic and probabilistic tractography based on complex fiber orientation distributions. Trans. on Med. Imag., 28(2):269-286, 2009.

39. Z. Ding, J. Gore, and A. Anderson. Classification and quantification of neuronal fiber pathways using diffusion tensor MRI. Magnetic Resonance in Medicine, 49:716-721, 2003.

40. S. Durrleman, P. Fillard, X. Pennec, A. Trouvé, and N. Ayache. A statistical model of white matter fiber bundles based on currents. In Information Processing in Medical Imaging (IPMI), pages 114-125, 2009.

41. I. Eckstein, D. W. Shattuck, J. L. Stein, K. L. McMahon, G. de Zubicaray, M. J. Wright, P. M. Thompson, and A. W. Toga. Active fibers: Matching deformable tract templates to diffusion tensor images. NeuroImage, 47:T82-T89, 2009.

42. D. B. Ennis, G. Kindlmann, I. Rodriguez, P. A. Helm, and E. R. McVeigh. Visualization of tensor fields using superquadric glyphs. Magnetic Resonance in Medicine, 53:169-176, 2005. 
43. P. Fillard, C. Poupon, and J.-F. Mangin. A novel global tractography algorithm based on an adaptive spin glass model. In Medical Image Computing and Computer Assisted Intervention (MICCAI), pages 927-934, 2009.

44. P. T. Fletcher and S. Joshi. Riemannian geometry for the statistical analysis of diffusion tensor data. Signal Processing, 87(2):250-262, 2007.

45. P. T. Fletcher, R. Tao, W. K. Joeng, and R. Whitaker. A volumetric approach to quantifying region-to-region white matter connectivity in diffusion tensor MRI. In Information Processing in Medical Imaging (IPMI), pages 346-358, 2007.

46. L. Frank. Characterization of anisotropy in high angular resolution diffusionweighted MRI. Magnetic Resonance in Medicine, 47:1083-1099, 2002.

47. R. Z. Freidlin, E. Özarslan, M. E. Komlosh, L.-C. Chang, C. G. Koay, D. K. Jones, and P. J. Basser. Parsimonious model selection for tissue segmentation and classification applications: A study using simulated and experimental DTI data. Trans. on Med. Imag., 26(11):1576-1584, 2007.

48. O. Friman, G. Farnebäck, and C.-F. Westin. A Bayesian approach for stochastic white matter tractography. Trans. on Med. Imag., 25(8):965-978, 2006.

49. X. Gigandet, P. Hagmann, M. Kurant, L. Cammoun, R. Meuli, and J.-P. Thiran. Estimating the confidence level of white matter connections obtained with MRI tractography. PLoS ONE, 3(12):e4006, 2008.

50. G. Gong, Y. He, L. Concha, C. Lebel, D. W. Gross, A. C. Evans, and C. Beaulieu. Mapping anatomical connectivity patterns of human cerebral cortex using in vivo diffusion tensor imaging tractography. Cerebral Cortex, 19(3):524-536, 2009.

51. C. B. Goodlett, P. T. Fletcher, J. H. Gilmore, and G. Gerig. Group analysis of DTI fiber tract statistics with application to neurodevelopment. NeuroImage, 45:S133-S142, 2009.

52. C. Gössl, L. Fahrmeir, B. P utz, L. Auer, and D. Auer. Fiber tracking from DTI using linear state space models: Detectability of the pyramidal tract. $\mathrm{Neu}$ roImage, 16:378-388, 2002.

53. W. Guo, Q. Zeng, Y. Chen, and Y. Liu. Using multiple tensor deflection to reconstruct white matter fiber traces with branching. In Int. Symp. on Biomedical Imaging, pages 69-72, 2006.

54. P. Hagmann. From Diffusion MRI to Brain Connectomics. PhD thesis, Université de Lausanne, 2005.

55. P. Hagmann, L. Cammoun, X. Gigandet, R. Meuli, C. J. Honey, V. J. Wedeen, and O. Sporns. Mapping the structural core of human cerebral cortex. PLoS Biology, 6(7):e159, 2008.

56. P. Hagmann, M. Kurant, X. Gigandet, P. Thiran, V. J. Wedeen, R. Meuli, and J. P. Thiran. Mapping human whole-brain structural networks with diffusion MRI. PLoS ONE, 2(7):e597, 2007.

57. P. Hagmann, T. Reese, W.-Y. Tseng, R. Meuli, J.-P. Thiran, and V. J. Wedeen. Diffusion spectrum imaging tractography in complex cerebral white matter: An investigation of the centrum semiovale. In Int. Symp. on Magnetic Resonance in Medicine (ISMRM), page 623, 2004.

58. C. Hess, P. Mukherjee, E. Han, D. Xu, and D. Vigneron. Q-ball reconstruction of multimodal fiber orientations using the spherical harmonic basis. Magnetic Resonance in Medicine, 56:104-117, 2006. 
59. C. J. Honey, O. Sporns, L. Cammoun, X. Gigandet, J. P. Thiran, R. Meulic, and P. Hagmann. Predicting human resting-state functional connectivity from structural connectivity. Proc. National Academy of Sciences, 106(6):2035-2040, 2009.

60. M. A. Horsfield and D. K. Jones. Neuropsychiatric applications of DTI - A review. NMR in Biomedicine, 15:587-593, 2002.

61. T. Hosey, G. Williams, and R. Ansorge. Inference of multiple fiber orientations in high angular resolution diffusion imaging. Magnetic Resonance in Medicine, 54:1480-1489, 2005.

62. K. Jansons and D. C. Alexander. Persistent angular structure: New insights from diffusion MRI data. Inverse Problems, 19:1031-1046, 2003.

63. S. Jbabdi, M. Woolrich, J. Andersson, and T. Behrens. A bayesian framework for global tractography. NeuroImage, 37:116-129, 2007.

64. B. Jian and B. Vemuri. A unified computational framework for deconvolution to reconstruct multiple fibers from diffusion weighted MRI. Trans. on Med. Imag., 26(11):1464-1471, 2007.

65. B. Jian, B. Vemuri, E. Özarslan, P. R. Carney, and T. H. Mareci. A novel tensor distribution model for the diffusion-weighted MR signal. NeuroImage, 37(1):164-176, 2007.

66. D. K. Jones. Determining and visualizing uncertainty in estimates of fiber orientation from diffusion tensor MRI. Magnetic Resonance in Medicine, 49:7$12,2003$.

67. D. K. Jones and C. Pierpaoli. Confidence mapping in diffusion tensor magnetic resonance imaging tractography using a bootstrap approach. Magnetic Resonance in Medicine, 53(5):1143-1149, 2005.

68. E. Kaden, T. Knøsche, and A. Anwander. Parametric spherical deconvolution: Inferring anatomical connectivity using diffusion MR imaging. NeuroImage, $37: 474-488,2007$.

69. G. Kindlmann, D. B. Ennis, R. T. Whitaker, and C.-F. Westin. Diffusion tensor analysis with invariant gradients and rotation tangents. Trans. on Med. Imag., 26(11):1483-1499, 2007.

70. G. Kindlmann, R. Estepar, M. Niethammer, S. Haker, and C.-F. Westin. Geodesic-loxodromes for diffusion tensor interpolation and difference measurement. In Medical Image Computing and Computer Assisted Intervention (MIC$C A I)$, pages $1-9,2007$.

71. C. G. Koay and P. J. Basser. Analytically exact correction scheme for signal extraction from noisy magnitude MR signals. J. of Magnetic Resonance, 179:317-322, 2006.

72. Martin A. Koch, David G. Norris, and Margret Hund-Georgiadis. An investigation of functional and anatomical connectivity using magnetic resonance imaging. NeuroImage, 16(1):241 - 250, 2002.

73. B. Kreher, I. Madeer, and V. Kiselev. Gibbs tracking: A novel approach for the reconstruction of neuronal pathways. Magnetic Resonance in Medicine, 60:953-963, 2008.

74. B. Kreher, J. Schneider, I. Mader, E. Martin, J. Hennig, and K. Il'yasov. Multitensor approach for analysis and tracking of complex fiber configurations. Magnetic Resonance in Medicine, 54:1216-1225, 2005.

75. M. Kubicki, R. McCarley, C.-F. Westin, H.-J. Park, S. Maier, R. Kikinis, F. A. Jolesz, and M. E. Shenton. A review of diffusion tensor imaging studies in schizophrenia. J. of Psychiatric Research, 41:15-30, 2007. 
76. S. Lankton, J. Melonakos, J. Malcolm, S. Dambreville, and A. Tannenbaum. Localized statistics for DW-MRI fiber bundle segmentation. In Mathematical Methods in Biomedical Image Analysis (MMBIA), pages 1-8, 2008.

77. M. Lazar and A. Alexander. Bootstrap white matter tractography (BootTract). NeuroImage, 24:524-532, 2005.

78. M. Lazar, D. M. Weinstein, J. S. Tsuruda, K. M. Hasan, K. Arfanakis, M. E. Meyerand, B. Badie, H. A Rowley, V. Haughton, A. Field, and A. L. Alexander. White matter tractography using diffusion tensor deflection. Human Brain Mapping, 18:306-321, 2003.

79. A. Leemans and D. K. Jones. A new approach to fully automated fiber tract clustering using affinity propagation. In Int. Symp. on Magnetic Resonance in Medicine (ISMRM), volume 17, page 856, 2009.

80. C. Lenglet, R. Deriche, and O. Faugeras. Inferring white matter geometry from diffusion tensor MRI: Application to connectivity mapping. In European Conf. on Computer Vision, pages 127-140, 2004.

81. K. Lim and J. Helpern. Applications of diffusion-weighted and diffusion tensor MRI to white matter diseases - a review. NMR in Biomedicine, 15:570-577, 2002.

82. C.-P. Lin, V. J. Wedeen, J.-H. Chen, C. Yao, and W.-Y. Tseng. Validation of diffusion spectrum magnetic resonance imaging with manganese-enhanced rat optic tracts and ex vivo phantoms. NeuroImage, 19:482-495, 2003.

83. M. Maddah, W. E. L. Grimson, S. K. Warfield, and W. M. Wells. A unified framework for clustering and quantitative analysis of white matter fiber tracts. Medical Image Analysis, 12(2):191-202, 2008.

84. J. Malcolm, Y. Rathi, and A. Tannenbaum. A graph cut approach to image segmentation in tensor space. In Component Analysis Methods (in CVPR), pages 1-8, 2007.

85. J. G. Malcolm, M. Kubicki, M. E. Shenton, and Y. Rathi. The effect of local fiber model on population studies. In Diffusion Modeling and Fiber Cup (in MICCAI), pages 33-40, 2009.

86. J. G. Malcolm, M. E. Shenton, and Y. Rathi. Filtered tractography: State estimation in a constrained subspace. In Diffusion Modeling and Fiber Cup (in MICCAI), pages 122-133, 2009.

87. J. G. Malcolm, M. E. Shenton, and Y. Rathi. Neural tractography using an unscented Kalman filter. In Information Processing in Medical Imaging (IPMI), pages 126-138, 2009.

88. T. McGraw, B. Vemuri, B. Yezierski, and T. Mareci. Von Mises-Fisher mixture model of the diffusion ODF. In Int. Symp. on Biomedical Imaging, pages 65-68, 2006.

89. L. Minati and W. P. Wȩogonglarz. Physical foundations, models, and methods of diffusion magnetic resonance imaging of the brain: A review. Concepts in Magnetic Resonance Part A, 30(5):278-307, 2007.

90. S. Mori and P. C. M. van Zijl. Fiber tracking: principles and strategies - A technical review. NMR in Biomedicine, 15:468-480, 2002.

91. D. G. Norris. The effects of microscopic tissue parameters on the diffusion weighted magnetic resonance imaging experiment. NMR in Biomedicine, 15:438-455, 2001.

92. L. O'Donnell, S. Haker, and C.-F. Westin. New approaches to estimation of white matter connectivity in diffusion tensor MRI: elliptic PDE's and geodesics 
in tensor-warped space. In Medical Image Computing and Computer Assisted Intervention (MICCAI), pages 459-466, 2002.

93. L. J. O'Donnell and C.-F. Westin. Automatic tractography segmentation using a high-dimensional white matter atlas. Trans. on Med. Imag., 26:1562-75, 2007.

94. L. J. O'Donnell, C.-F. Westin, and A. J. Golby. Tract-based morphometry for white matter group analysis. NeuroImage, 45(3):832-844, 2009.

95. E. Özarslan and T. Mareci. Generalized diffusion tensor imaging and analytical relationships between diffusion tensor imaging and high angular resolution diffusion imaging. Magnetic Resonance in Medicine, 50:955-965, 2003.

96. E. Özarslan, T. Shepherd, B. Vemuri, S. Blackband, and T. Mareci. Resolution of complex tissue microarchitecture using the diffusion orientation transform. NeuroImage, 31(3):1086-1103, 2006.

97. E. Özarslan, B. C. Vemuri, and T. H. Mareci. Generalized scalar measures for diffusion MRI using trace, variance, and entropy. Magnetic Resonance in Medicine, 53:866-876, 2005.

98. G. Parker and D. C. Alexander. Probabilistic Monte Carlo based mapping of cerebral connections utilizing whole-brain crossing fiber information. In Information Processing in Medical Imaging (IPMI), pages 684-696, 2003.

99. G. Parker and D. C. Alexander. Probabilistic anatomical connectivity derived from the microscopic persistent angular structure of cerebral tissue. Phil. Trans. R. Soc. B, 360:893-902, 2005.

100. S. Peled, O. Friman, F. Jolesz, and C.-F. Westin. Geometrically constrained two-tensor model for crossing tracts in DWI. Magnetic Resonance in Medicine, 24(9):1263-1270, 2006.

101. M. Perrin, C. Poupon, Y. Cointepas, B. Rieul, N. Golestani, C. Pallier, D. Riviere, A. Constantinesco, D. Le Bihan, and J.-F. Mangin. Fiber tracking in Q-ball fields using regularized particle trajectories. In Information Processing in Medical Imaging (IPMI), pages 52-63, 2005.

102. E. Pichon, C.-F. Westin, and A. Tannenbaum. A hamilton-jacobi-bellman approach to high angular resolution diffusion tractography. In Medical Image Computing and Computer Assisted Intervention (MICCAI), pages 180-187, 2005.

103. K. M. Pohl, R. Kikinis, and W. M. Wells. Active mean fields: Solving the mean field approximation in the level set framework. In Information Processing in Medical Imaging (IPMI), pages 26-37, 2007.

104. C. Poupon, B. Rieul, I. Kezele, M. Perrin, F. Poupon, and J. F. Mangin. New diffusion phantoms dedicated to the study and validation of highangular-resolution diffusion imaging (HARDI) models. Magnetic Resonance in Medicine, 60(6):1276-83, 2008.

105. C. Poupon, A. Roche, J. Dubois, J.-F. Mangin, and F. Poupon. Real-time MR diffusion tensor and Q-ball imaging using Kalman filtering. Medical Image Analysis, 12(5):527-534, 2008.

106. E. Prados, C. Lenglet, J. Pons, N. Wotawa, R. Deriche, O. Faugeras, and S. Soatto. Control theory and fast marching techniques for brain connectivity mapping. In Computer Vision and Pattern Recognition (CVPR), pages 10761083, 2006.

107. Y. Rathi, O. Michailovich, M. E. Shenton, and S. Bouix. Directional functions for orientation distribution estimation. Medical Image Analysis, 13:432-444, 2009 . 
108. Y. Rathi, O. Michailovich, and A. Tannenbaum. Segmenting images on the tensor manifold. In Computer Vision and Pattern Recognition (CVPR), 2007.

109. M. Reisert, I. Mader, and V. Kiselev. Global reconstruction of neuronal fibres. In Diffusion Modeling and Fiber Cup (in MICCAI), pages 70-81, 2009.

110. T. Rohlfing, E. V. Sullivan, and A. Pfefferbaum. Regression models of atlas appearance. In Information Processing in Medical Imaging (IPMI), pages 151$162,2009$.

111. M. Rousson, C. Lenglet, and R. Deriche. Level set and region based propagation for diffusion tensor MRI segmentation. In ECCV, Workshops CVAMIAMMBIA, 2004.

112. P. Savadjiev, J. Campbell, G. Pike, and K. Siddiqi. 3D curve inference for diffusion MRI regularization and fibre tractography. Medical Image Analysis, 10:799-813, 2006.

113. P. Savadjiev, J. S. Campbell, M. Descoteaux, R. Deriche, G. B. Pike, and K. Siddiqi. Labeling of ambiguous subvoxel fibre bundle configurations in high angular resolution diffusion MRI. NeuroImage, 41:58-68, 2008.

114. P. Savadjiev, G. Kindlmann, S. Bouix, M. E. Shenton, and C.-F. Westin. Local white matter geometry indices from diffusion tensor gradients. In Medical Image Computing and Computer Assisted Intervention (MICCAI), pages 345352, 2009.

115. T. Schultz and H. Seidel. Estimating crossing fibers: A tensor decomposition approach. Trans. on Visualization and Computer Graphics, 14(6):1635-1642, 2008.

116. S. M. Smith, M. Jenkinson, H. Johansen-Berg, D. Rueckert, T. E Mackay, K. E. Watkins, O. Ciccarelli, M. Z. Cader, P. M. Matthews, and T. E. Behrens. Tractbased spatial statistics: Voxelwise analysis of multi-subject diffusion data. NeuroImage, 31:1487-1505, 2006.

117. O. Sporns, C. J. Honey, and R. Kötter. Identification and classification of hubs in brain networks. PLoS ONE, 2(10):e1049, 2007.

118. J.-D. Tournier, F. Calamante, and A. Connelly. Robust determination of the fibre orientation distribution in diffusion MRI: Non-negativity constrained super-resolved spherical deconvolution. NeuroImage, 35:1459-1472, 2007.

119. J.-D. Tournier, F. Calamante, D. Gadian, and A. Connelly. Diffusion-weighted magnetic resonance imaging fibre tracking using a front evolution algorithm. NeuroImage, 20:276-288, 2003.

120. J.-D. Tournier, F. Calamante, D. Gadian, and A. Connelly. Direct estimation of the fiber orientation density function from diffusion-weighted MRI data using spherical deconvolution. NeuroImage, 23:1176-1185, 2004.

121. D. Tschumperlé. PDE-Based Regularization of Multivalued Images and Applications. PhD thesis, University of Nice-Sophia Antipolis, 2002.

122. D. Tuch. Diffusion MRI of Complex Tissue Structure. PhD thesis, Massachusetts Institute of Technology, 2002.

123. D. Tuch. Q-ball imaging. Magnetic Resonance in Medicine, 52:1358-1372, 2004.

124. D. Tuch, J. Belliveau, and V. J. Wedeen. A path integral approach to white matter tractography. In Int. Symp. on Magnetic Resonance in Medicine (ISMRM), page 791, 2000.

125. D. Tuch, T. Reese, M. Wiegell, N Makris, J. Belliveau, and V. Wedeen. High angular resolution diffusion imaging reveals intravoxel white matter fiber heterogeneity. Magnetic Resonance in Medicine, 48:577-582, 2002. 
126. A. Vilanova, S. Zhang, G. Kindlmann, and D. Laidlaw. An introduction to visualization of diffusion tensor imaging and its applications. In Visualization and Processing of Tensor Fields, chapter 7, pages 121-153. Springer, 2006.

127. X. Wang, W. E. L. Grimson, and C.-F. Westin. Tractography segmentation using a hierarchical dirichlet processes mixture model. In Information Processing in Medical Imaging (IPMI), pages 101-113, 2009.

128. D. Wassermann, L. Bloy, E. Kanterakis, R. Verma, and R. Deriche. Unsupervised white matter fiber clustering and tract probability map generation: Applications of a gaussian process framework for white matter fibers. Technical Report 7005, INRIA, 2009.

129. V. J. Wedeen, P. Hagmann, W.-Y. Tseng, T. Reese, and R. M. Weisskoff. Mapping complex tissue architecture with diffusion spectrum magnetic resonance imaging. Magnetic Resonance in Medicine, 54:1377-1386, 2005.

130. C.-F. Westin, S. Maier, H. Mamata, A. Nabavi, F. Jolesz, and R. Kikinis. Processing and visualizationfor diffusiontensor MRI. Medical Image Analysis, 6:93-108, 2002.

131. B. Whitcher, D. Tuch, J. Wisco, A. Sorensen, and L. Wang. Using the wild bootstrap to quantify uncertainty in diffusion tensor imaging. Human Brain Mapping, 29(3):346-362, 2008.

132. P. A. Yushkevich, H. Zhang, T. J. Simon, and J. C. Gee. Structure-specific statistical mapping of white matter tracts. NeuroImage, 41:448-461, 2008.

133. F. Zhang, E. Hancock, C. Goodlett, and G. Gerig. Probabilistic white matter fiber tracking using particle filtering and von Mises-Fisher sampling. Medical Image Analysis, 13:5-18, 2009.

134. L. Zhukov and A. Barr. Oriented tensor reconstruction: Tracing neural pathways from diffusion tensor MRI. In Visualization, pages 387-394, 2002. 